\title{
Dirty living breeds better immune health
}

Conventional wisdom holds that exposure to dirt and germs is bad for one's health: after all, 'cleanliness is next to godliness.' More recently, the high prevalence of immune-mediated diseases in industrialized countries (with relatively high standards for cleanliness and sanitation) has called this notion into question. The 'hygiene hypothesis' proposes that the increasing incidence of immune-related disorders results from reduced exposure to microbes in early childhood. Indeed, previous research has shown that animals that are susceptible to autoimmune disease are more likely to develop disease (and their disease is more severe) when they are raised in germ-free conditions than when they are exposed to bacteria. Now, a new study by Denise Kelly (University of Aberdeen, UK) and colleagues establishes a direct link between microbe exposure and immune health.

Kelly's study assessed the effects of early life environment on gut microbes and immune health in piglets. Piglets were housed in one of three environments: outdoors, indoors or in isolators. Piglets

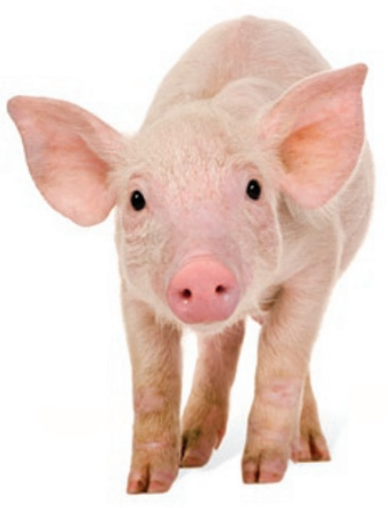

Escherichia coli and salmonella in the gut. In contrast, piglets that were housed indoors in hygienic conditions had fewer Lactobacillus and more potentially pathogenic bacteria.

The researchers also examined the expression of genes associated with immune function in the three groups of piglets. In piglets housed in isolators, expression of genes involved in inflammatory immune responses and cholesterol synthesis was elevated. In piglets housed outdoors, however, expression of genes associated with $\mathrm{T}$ cells was elevated. These results suggest that the piglets housed outdoors had a more stable and tolerant immune system than did those housed indoors.

This study is the first to demonstrate a causal link between living environment and immune response. Although it is not known how closely the piglets' physiology represents human physiology, Kelly believes that the similarities in the microbial communities of pigs and humans and their comparable sizes make pigs a good model.

Monica Harrington

\section{HELPTNG INHURED RATS REGATN MOVEMENT}

In January 2009, the US Food and Drug Administration (FDA) approved the first human trial of an embryonic stem cell-based treatment. Led by the biotech company Geron (Menlo Park, California), researchers plan to test this therapy in people who have become paralyzed from the waist down due to acute thoracic (back) spinal cord injuries. The aim of the therapy, called GRNOPC1, is to help these patients regain some sensation and movement in their lower limbs.

About $48 \%$ of acute spinal injuries in humans are thoracic injuries, while $52 \%$ are cervical (neck) injuries. Due to a lack of animal data, the FDA has not approved the therapy for testing in humans with acute cervical injuries. However, researchers have now reported that this therapy helps rats with acute cervical spinal injuries.

GRNOPC1 consists of oligodendrocyte progenitor cells that are derived from human embryonic stem cells. These cells become oligodendrocytes, which form myelin, an insulating material that helps speed up nerve cell communication. The GRNOPC1 therapy traces its roots back to work done by Hans Keirstead of the University of California at Irvine and his colleagues. In 2005 Keirstead and his team reported that the therapy helped restore movement in rats with thoracic injuries (J. Neurosci., 25, 4694-4705; 2005). Now, doctoral student Jason Sharpe, Keirstead and colleagues have shown that the oligodendrocyte progenitor cell therapy helped restore motor function in rats with acute cervical spinal cord injuries (Stem Cells published online 28 October, 2009; doi:10.1002/stem.245).

The research team injured spinal cords of adult female rats at the cervical level. One week later, they injected the oligodendrocyte progenitor cell product into the spinal cords of half of the rats and a control solution into the remaining rats. They tested forelimb motor function of both groups of rats and found that the injections significantly improved forelimb motor function. Further analysis showed that injecting the oligodendrocyte progenitor cells into the injured rats helped to rebuild myelin, prevented tissue death and even prompted nerve fiber regrowth.

In August, the FDA put the GRNOPC1 trial for patients with acute thoracic injuries on hold. Pending FDA approval of additional animal data that will be submitted by Geron, the thoracic trial should be re-initiated in spring 2010 . Keirstead and colleagues hope their recent results will make it more likely for the FDA to allow the therapy to also be tested in people with acute cervical injuries.

Kirsten Dorans 